\title{
Evaluation of the Relevant Environmental Aspects of Some Locations Intended for New Investments
}

\author{
BOGDAN STANESCU*, LIDIA KIM, GINA TRAISTARU, ADRIANA CUCIUREANU, GHEORGHITA TANASE \\ National Research and Development Institute for Industrial Ecology, ECOIND, 71-73, Drumul Podu Dambovitei Str., 060652, \\ Bucharest, Romania
}

\begin{abstract}
In recent years, the growing dynamics of the investments for new civil and industrial construction projects involves new properties acquisitions. On the other hand, there is an increase in the conversion of some industrial sites into commercial or residential areas, after their acquisition by the new owners. It is very important to knows the quality of the environmental components in the areas where the new investment projects are being implemented. Most of the time, the acquisition of a land or industrial site is preceded and, in many cases, conditioned by a thorough assessment of the quality of the environmental components in order to assume responsibilities for the ensuing environmental obligations. This involve environmental assessment performed by specialists who can objectively and analyze relevant environmental issues and report the necessary conclusions and recommendations for each case. The paper presents the expertise of the National Research and Development Institute for Industrial Ecology specialists in a study that analyzed soil, groundwater and air quality in order to develop new investments projects. The presented conclusions converge towards the important role of the environmental assessment based on the relevant information analyzed for each case and the importance of environmental information in preparing new investments.
\end{abstract}

Keywords: environmental assessment, pollution sources, potential pollution.

The environmental quality of a site involves a set of general and local environmental and physical parameters that can affect humans and other organisms. A primordial aspect is given by human health that includes the quality of life that is equivalent to a clean, unpolluted environment [1-2].

Air quality is determined by emissions from stationary and mobile sources, as long as long-distance pollutant transport by wind in synergism with other factors [3-4]. All environmental factor from air to soil, groundwater are very important in a complex and objective environmental assessment [5-7]. The development needs of Bucharest, the capital of Romania are very high, but but at the same time is considered one of the polluted cities [8]. In recent years, the interest of large investment firms in the construction of industrial buildings, logistic bases (warehouses) and offices has been noted. One of these companies present on the Romanian market has been oriented towards providing the clients with facilities in the transit areas, but not so far from the public transport facilities of Bucharest city. This international company has built and builds facilities, warehouses for adequate storage of vegetables, fruits, for electronics and household appliances, medicines, etc. All facilities are built to the highest quality standards and also offers office buildings for its clients in accordance with their needs [9].

To develop these facilities, they acquired a number of lands that were set aside and underwent major transformations being converted into industrial or commercial facilities.

The environmental protection developer wanted to know in detail the quality of the environmental components for the purchased land so as to ensure that all the environmental requirements covered by the Romanian legislation are meet. National Research and Development Institute for Industrial Ecology specialists made an objective assessment of the quality of the environmental components and submitted the conclusions of the investigations to the developer, assuring them that there are no prerequisites with a negative potential for the implementation of their development projects.

\section{Experimental part}

The analyzed site was a land located near the city of Bucharest, in its Westarea, in the proximity of a road junction

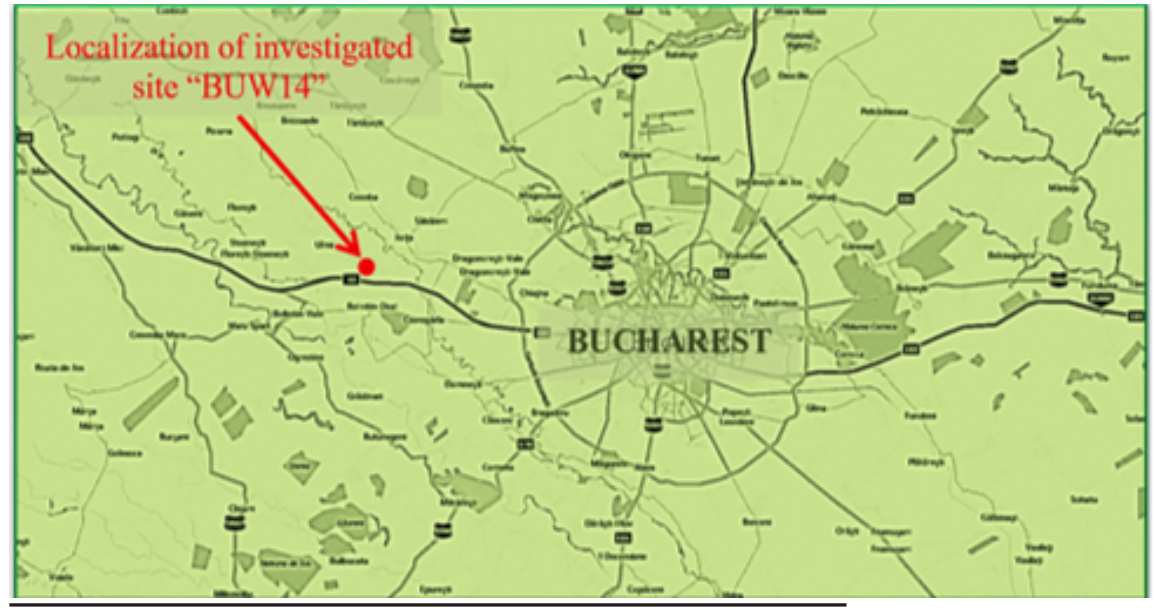

*email: stanescubogdan2@gmail.com
Fig. 1. Localization of the investigated site BUW 14 
located on the A1 highway, Bucharest-Pitesti, km. 23, exit to Ghionea village, respectively on secondary road DC147.

The land area is about 10 hectares and was coded under the BUW 14 label. The localization of the area investigated is shown in figure 1.

The investigations aimed to make a complete analysis of the environmental factors, for a more thorough assessment in order to establish the initial conditions, respectively before starting the investment project in this zone. The proposed investigations for the BUW 14 site are presented schematically in figure 2.

Air and soil quality investigation points can be seen in figures 3 and 4 . The noise measurements were performed at the same points as air quality investigations.

Specific sampling and measuring equipmentwere used for different types of samples: autolaboratory for air

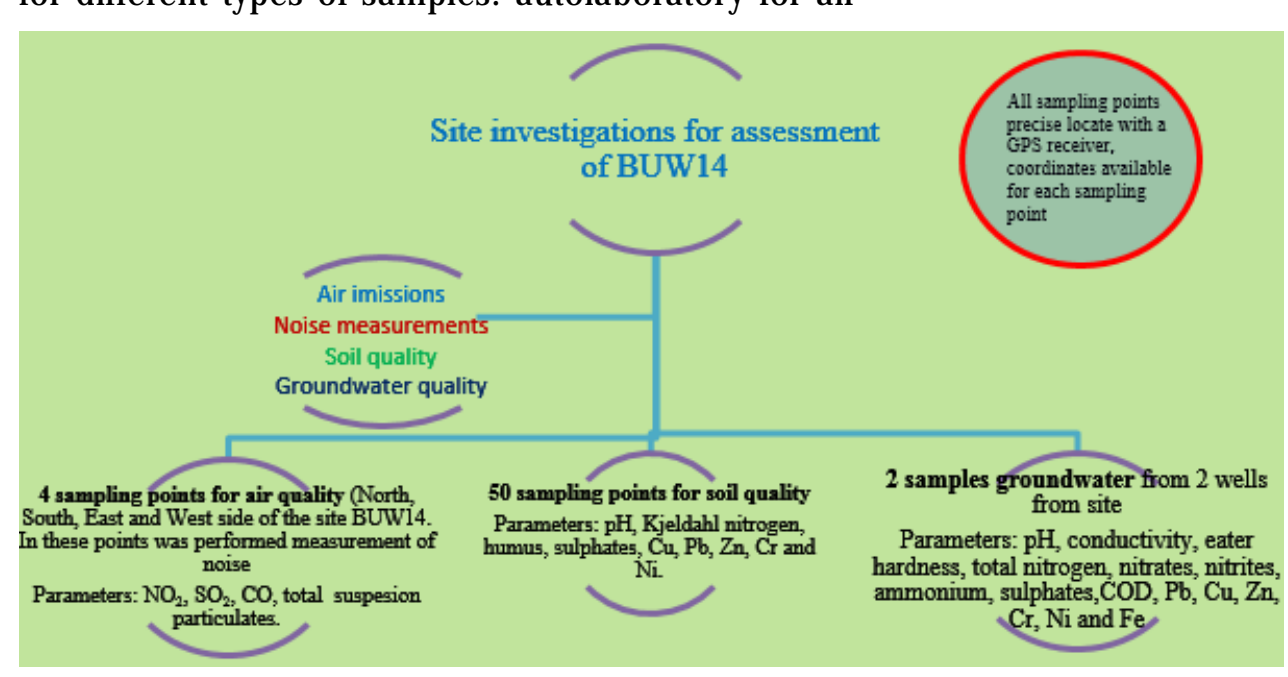

measurements, hand operated auger sistem (Eijkelkamp) for soil sampling, electric pump for two wells groundwater sampling existing on BUW14 site.

The samples were uniquely labeled, properly preserved and transported on the same day as the sampling (29/11/ 2017) at the National Research and Development Institute for Industrial Ecology Laboratories. For all tests have been applied standardized analytical methods, the equipment used beeing metrologicaly controled and calibrated with certified reference materials.

\section{Results and discussions}

Level of immissions

The results for chemical pollutants in air are presented in table 1.

Fig. 2. Site investigations data sheet BUW 14

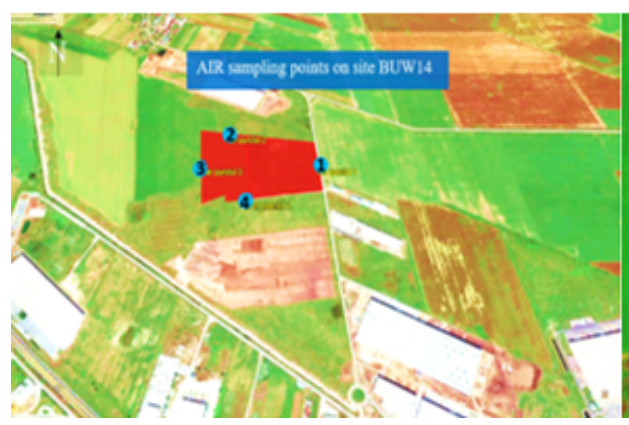

Fig. 3. Air sampling points locations

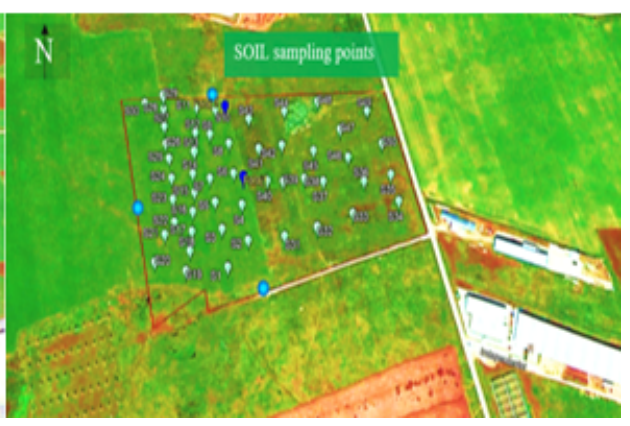

Fig. 4. Soil sampling points locations

Table 1

AIR QUALITY RESULTS

\begin{tabular}{|c|c|c|c|c|}
\hline \multirow{2}{*}{$\begin{array}{c}\text { Air sampling } \\
\text { points }\end{array}$} & \multicolumn{4}{|c|}{ Pollutant concentrations } \\
\cline { 2 - 5 } & $\mathrm{TSP}, \mathrm{mg} / \mathrm{mc}$ & $\mathrm{NO}_{2}, \mu \mathrm{g} / \mathrm{mc}$ & $\mathrm{SO}_{2}, \mu \mathrm{g} / \mathrm{mc}$ & $\mathrm{CO}, \mu \mathrm{g} / \mathrm{mc}$ \\
\hline 1 & 0.19 & 106 & 9.3 & 0.46 \\
\hline 2 & 0.14 & 68.3 & 8.1 & 0.32 \\
\hline 3 & 0.15 & 70.6 & 7.6 & 0.39 \\
\hline 4 & 0.17 & 92.3 & 8.9 & 0.42 \\
\hline Limit Values & 0.50 & 200 & 350 & 10,000 \\
\hline
\end{tabular}

Table 2

RESULTS OF NOISE LEVEL MEASUREMENTS

\begin{tabular}{|c|c|c|c|c|c|}
\hline Measuring point & Noise type & $\mathbf{L}_{\text {eq }}, \mathbf{d B}(\mathbf{A})$ & Measuring point & Noise type & $\mathbf{L}_{\text {eq }}, \mathbf{d B}(\mathbf{A})$ \\
\hline 1 & day & 54.3 & 3 & day & 48.6 \\
\hline 2 & day & 49.3 & 4 & day & 56.3 \\
\hline
\end{tabular}


Analyzing the results of the measurements performed at all four sampling points located at the boundary of the field BUW 14, compared with the maximum admissible values from in force legislation $[10,11]$, it was found that the values of the measured concentrations of the chemical pollutants were below the Limit Values.

The noise level

Regarding the noise level, the results obtained with a digital sound-meter are presented in table 2 . The measured values for the noise level at the enclosure limit were situated below $65 \mathrm{~dB}(\mathrm{~A})$, which represent the admitted limit value [12].

\section{Soil quality}

Tables 3 $\div 7$ presents the analytical results obtained from the characterization of the 50 soil samples, colected from first soil layer, up to 15 centimetrs depth $[13,14]$.

The morphological analysis of soil samples taken from the BUW14 field reveals a textural homogeneity, the soils identified correspond to the pedogenetic loess formations specific to the area in the proximity of the municipality of Bucharest.

\begin{tabular}{|c|c|c|c|c|c|c|c|c|c|c|c|c|c|}
\hline \multirow[t]{2}{*}{ Parameter } & \multirow[t]{2}{*}{ S1 } & \multirow[t]{2}{*}{ S2 } & \multirow[t]{2}{*}{ S3 } & \multirow[t]{2}{*}{ S4 } & \multirow[t]{2}{*}{ S5 } & \multirow[t]{2}{*}{ S6 } & \multirow[t]{2}{*}{ S7 } & \multirow[t]{2}{*}{ S8 } & \multirow[t]{2}{*}{ S9 } & \multirow[t]{2}{*}{$\mathrm{S} 10$} & \multicolumn{3}{|c|}{$\begin{array}{c}\text { Reference values from } \\
\text { Order } 756 / 1997, \\
\text { less sensinive land use }\end{array}$} \\
\hline & & & & & & & & & & & 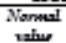 & 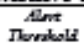 & $\begin{array}{l}\text { Thamentain } \\
\text { Muratele }\end{array}$ \\
\hline $\mathrm{pH}$ (pH units) & 8.8 & 8.6 & 8.5 & 8 & 7.5 & 7.1 & 7.5 & 7 & 7.4 & 7.3 & - & - & - \\
\hline Dry matter $(\%)$ & 88.3 & 89.3 & 91.8 & 90.0 & 88.4 & 85.8 & 89.9 & 92.9 & 89.5 & 89.9 & - & - & - \\
\hline Humus $(\% \mathrm{dm})$ & 0.65 & 2.9 & 0.66 & 2.78 & 0.68 & 4.76 & 4.42 & 2.47 & 2.62 & 2.40 & - & - & - \\
\hline $\begin{array}{l}\text { Sulphates (mg/kg } \\
\mathrm{dm} \text { ) }\end{array}$ & 453 & 325 & 260 & 275 & 226 & 315 & 334 & 390 & 420 & 375 & - & 5,000 & 50,000 \\
\hline $\begin{array}{l}\text { Kjeldahl Nitrogen } \\
(\% \mathrm{dm})\end{array}$ & 0.14 & 0.14 & 0.14 & 0.16 & 0.16 & 0.14 & 0.14 & 0.13 & 0.14 & 0.14 & - & - & - \\
\hline $\mathrm{Cu}(\mathrm{mg} / \mathrm{kg} \mathrm{dm})$ & 20.4 & 18.0 & 19.9 & 20.8 & 20.2 & 20.9 & 20.2 & 20.6 & 21.5 & 22.5 & 20 & 250 & 500 \\
\hline $\mathrm{Zn}(\mathrm{mg} / \mathrm{kg} \mathrm{dm})$ & 55.6 & 57.4 & 57.3 & 59.2 & 57.0 & 61.3 & 59.2 & 58.1 & 61.2 & 59.4 & 100 & 700 & 1,500 \\
\hline $\mathrm{Cr}(\mathrm{mg} / \mathrm{kg} \mathrm{dm})$ & 0.79 & 0.78 & 0.77 & 0.80 & 0.78 & 1.12 & 0.73 & 0.82 & 0.83 & 0.88 & 30 & 300 & 600 \\
\hline $\mathrm{Pb}(\mathrm{mg} / \mathrm{kg} \mathrm{dm})$ & 22.3 & 20.4 & 20.4 & 20.0 & 20.5 & 23.0 & 19.8 & 22.1 & 21.6 & 22.1 & 20 & 250 & 1,000 \\
\hline $\mathrm{Ni}(\mathrm{mg} / \mathrm{kg} \mathrm{dm})$ & 33.6 & 34.5 & 33.2 & 33.2 & 34.9 & 31.5 & 33.1 & 32.5 & 34.6 & 36.0 & 20 & 200 & 500 \\
\hline
\end{tabular}

Table 3

SOIL RESULTS FOR S1 $\div$ S10 SOIL SAMPLES FROM BUW14 SITE

\begin{tabular}{|c|c|c|c|c|c|c|c|c|c|c|c|c|c|}
\hline \multirow[t]{2}{*}{ Parameter } & \multirow[t]{2}{*}{ S11 } & \multirow[t]{2}{*}{ S12 } & \multirow[t]{2}{*}{ S13 } & \multirow[t]{2}{*}{ S14 } & \multirow[t]{2}{*}{ S15 } & \multirow[t]{2}{*}{ S16 } & \multirow[t]{2}{*}{ S17 } & \multirow[t]{2}{*}{ S18 } & \multirow[t]{2}{*}{ S19 } & \multirow[t]{2}{*}{$\mathrm{S} 20$} & \multicolumn{3}{|c|}{$\begin{array}{l}\text { Reference values from } \\
\text { Order } 756 / 1997, \\
\text { less sensinive land use }\end{array}$} \\
\hline & & & & & & & & & & & $\begin{array}{c}\text { Nonnelal } \\
\text { vihu }\end{array}$ & Thents & 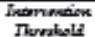 \\
\hline $\mathrm{pH}$ (pH units) & 7.5 & 6.8 & 7 & 6.8 & 7.8 & 7.7 & 7.3 & 7.1 & 7.2 & 7.3 & - & - & - \\
\hline Dry matter (\%) & 88.6 & 88.8 & 90.6 & 90.3 & 88.6 & 88.7 & 90.5 & 87.8 & 90.5 & 87.8 & - & - & - \\
\hline Humus $(\% \mathrm{dm})$ & 3.18 & 2.94 & 2.77 & 2.80 & 3.54 & 3.47 & 2.95 & 3.79 & 2.36 & 2.34 & - & - & - \\
\hline $\begin{array}{l}\text { Sulphates }(\mathrm{mg} / \mathrm{kg} \\
\mathrm{dm})\end{array}$ & 239 & 255 & 305 & 312 & 334 & 255 & 296 & 297 & 296 & 315 & - & 5,000 & 50,000 \\
\hline $\begin{array}{l}\text { Kjeldahl Nitrogen } \\
(\% \mathrm{dm})\end{array}$ & 0.14 & 0.13 & 0.14 & 0.14 & 0.16 & 0.19 & 0.15 & 0.14 & 0.14 & 0.14 & - & - & - \\
\hline $\mathrm{Cu}(\mathrm{mg} / \mathrm{kg} \mathrm{dm})$ & 21.8 & 19.8 & 20.2 & 22.4 & 16.7 & 20.5 & 19.7 & 13.2 & 18.8 & 20.3 & 20 & 250 & 500 \\
\hline $\mathrm{Zn}(\mathrm{mg} / \mathrm{kg} \mathrm{dm})$ & 57.1 & 56.5 & 53.7 & 55.9 & 50.7 & 54.6 & 52.5 & 38.7 & 48.4 & 58.1 & 100 & 700 & 1,500 \\
\hline $\mathrm{Cr}(\mathrm{mg} / \mathrm{kg} \mathrm{dm})$ & 0.80 & 0.77 & 0.79 & 0.90 & 0.38 & 0.79 & 0.83 & 0.37 & 0.71 & 0.78 & 30 & 300 & 600 \\
\hline $\mathrm{Pb}(\mathrm{mg} / \mathrm{kg} \mathrm{dm})$ & 21.9 & 19.6 & 22.1 & 21.3 & 13.8 & 20.2 & 19.5 & 10.8 & 18.9 & 18.8 & 20 & 250 & 1,000 \\
\hline $\mathrm{Ni}(\mathrm{mg} / \mathrm{kg} \mathrm{dm})$ & 32.5 & 33.1 & 33.3 & 36.3 & 30.9 & 31.1 & 27.1 & 35.2 & 35.5 & 33.8 & 20 & 200 & 500 \\
\hline
\end{tabular}

\begin{tabular}{|c|c|c|c|c|c|c|c|c|c|c|c|c|c|}
\hline \multirow[t]{2}{*}{ Parameter } & \multirow[t]{2}{*}{ S21 } & \multirow[t]{2}{*}{ S22 } & \multirow[t]{2}{*}{ S23 } & \multirow[t]{2}{*}{ S24 } & \multirow[t]{2}{*}{ S25 } & \multirow[t]{2}{*}{ S26 } & \multirow[t]{2}{*}{ S27 } & \multirow[t]{2}{*}{ S28 } & \multirow[t]{2}{*}{ S29 } & \multirow[t]{2}{*}{$\mathrm{s} 30$} & \multicolumn{3}{|c|}{$\begin{array}{c}\text { Reference values from } \\
\text { Order } 756 / 1997 \text {, } \\
\text { less sensitive land use }\end{array}$} \\
\hline & & & & & & & & & & & $\begin{array}{c}\text { Normel } \\
\text { vihu }\end{array}$ & Thentels & 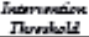 \\
\hline $\mathrm{pH}$ (pH units) & 6.7 & 6.6 & 6.7 & 6.6 & 6.8 & 7 & 6.9 & 6.4 & 7 & 6.6 & - & - & - \\
\hline Dry matter (\%) & 86.3 & 89.8 & 92.2 & 92.2 & 91.9 & 91.7 & 88.2 & 89.3 & 90.8 & 96.4 & - & - & - \\
\hline Humus $(\% \mathrm{dm})$ & 2.01 & 1.58 & 3.56 & 3.38 & 2.51 & 2.28 & 2.80 & 3.10 & 2.39 & 2.88 & - & - & - \\
\hline $\begin{array}{l}\text { Sulphates (mg/kg } \\
\mathrm{dm})\end{array}$ & 452 & 395 & 325 & 318 & 309 & 290 & 227 & 275 & 332 & 339 & - & 5,000 & $\mathbf{5 0 , 0 0 0}$ \\
\hline $\begin{array}{l}\text { Kjeldahl Nitrogen } \\
(\% \mathrm{dm})\end{array}$ & 0.14 & 0.14 & 0.13 & 0.15 & 0.15 & 0.15 & 0.15 & 0.16 & 0.13 & 0.14 & - & - & - \\
\hline $\mathrm{Cu}(\mathrm{mg} / \mathrm{kg} \mathrm{dm})$ & 20.0 & 20.7 & 21.4 & 11.8 & 20.0 & 20.8 & 20.2 & 20.1 & 20.0 & 17.5 & 20 & 250 & 500 \\
\hline $\mathrm{Zn}(\mathrm{mg} / \mathrm{kg} \mathrm{dm})$ & 58.6 & 56.8 & 56.9 & 36.9 & 53.6 & 54.2 & 55.7 & 54.0 & 54.0 & 48.2 & 100 & 700 & 1,500 \\
\hline $\mathrm{Cr}_{\mathrm{r}}(\mathrm{mg} / \mathrm{kg} \mathrm{dm})$ & 0.77 & 0.87 & 0.89 & 0.26 & 0.78 & 0.84 & 0.79 & 0.76 & 0.76 & 0.76 & 30 & 300 & 600 \\
\hline $\mathrm{Pb}(\mathrm{mg} / \mathrm{kg} \mathrm{dm})$ & 20.8 & 20.3 & 19.9 & 9.3 & 18.8 & 22.1 & 21.7 & 19.6 & 19.8 & 18.3 & 20 & 250 & 1,000 \\
\hline $\mathrm{Ni}(\mathrm{mg} / \mathrm{kg} \mathrm{dm})$ & 34.6 & 37.4 & 31.1 & 30.6 & 30.2 & 30.7 & 33.5 & 32.2 & 32.3 & 31.9 & 20 & 200 & 500 \\
\hline
\end{tabular}

\begin{tabular}{|c|c|c|c|c|c|c|c|c|c|c|c|c|c|}
\hline \multirow[t]{2}{*}{ Parameter } & \multirow[t]{2}{*}{ S31 } & \multirow[t]{2}{*}{ S32 } & \multirow[t]{2}{*}{ S33 } & \multirow[t]{2}{*}{ S34 } & \multirow[t]{2}{*}{ S35 } & \multirow[t]{2}{*}{ S36 } & \multirow[t]{2}{*}{ S37 } & \multirow[t]{2}{*}{ S38 } & \multirow[t]{2}{*}{ S39 } & \multirow[t]{2}{*}{$\mathrm{S} 40$} & \multicolumn{3}{|c|}{$\begin{array}{c}\text { Reference values from } \\
\text { Order } 756 / 1997 \\
\text { less sensitive land use }\end{array}$} \\
\hline & & & & & & & & & & & $\begin{array}{ll}\text { Narnal } \\
\text { vhum }\end{array}$ & ALmo & 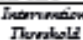 \\
\hline $\mathrm{pH}$ (pH units) & 7.6 & 8.1 & 8.4 & 7.2 & 7.5 & 7.8 & 7.1 & 7.5 & 6.9 & 7.8 & - & - & - \\
\hline Dry matter (\%) & 89.5 & 94.8 & 87.4 & 92.3 & 86.5 & 88.7 & 92.5 & 93.7 & 88.5 & 92.6 & - & - & - \\
\hline Humus $(\% \mathrm{dm})$ & 4.31 & 3.64 & 3.81 & 4.18 & 4.28 & 4.45 & 2.43 & 2.68 & 2.33 & 2.18 & - & - & - \\
\hline $\begin{array}{l}\text { Sulphates }(\mathrm{mg} / \mathrm{kg} \\
\mathrm{dm})\end{array}$ & 335 & 385 & 439 & 495 & 521 & 395 & 325 & 350 & 321 & 340 & - & 5,000 & $\mathbf{5 0 , 0 0 0}$ \\
\hline $\begin{array}{l}\text { Kjeldahl Nitrogen } \\
(\% \mathrm{dm})\end{array}$ & 0.14 & 0.14 & 0.16 & 0.15 & 0.15 & 0.14 & 0.15 & 0.15 & 0.15 & 0.14 & - & - & - \\
\hline $\mathrm{Cu}(\mathrm{mg} / \mathrm{kg} \mathrm{dm})$ & 24.4 & 22.5 & 25.7 & 25.0 & 26.3 & 23.4 & 26.3 & 27.3 & 27.9 & 27.5 & 20 & 250 & 500 \\
\hline $\mathrm{Zn}(\mathrm{mg} / \mathrm{kg} \mathrm{dm})$ & 59.3 & 54.0 & 69.2 & 65.7 & 69.8 & 66.0 & 68.8 & 67.5 & 68.8 & 66.8 & 100 & 700 & 1,500 \\
\hline $\mathrm{Cr}(\mathrm{mg} / \mathrm{kg} \mathrm{dm})$ & 0.72 & 0.78 & 0.87 & 0.91 & 0.80 & 0.74 & 0.82 & 0.88 & 0.86 & 0.90 & 30 & 300 & 600 \\
\hline $\mathrm{Pb}(\mathrm{mg} / \mathrm{kg} \mathrm{dm})$ & 21.6 & 20.7 & 23.2 & 20.9 & 24.4 & 20.2 & 19.8 & 18.7 & 20.4 & 19.2 & 20 & 250 & 1,000 \\
\hline $\mathrm{Ni}(\mathrm{mg} / \mathrm{kg} \mathrm{dm})$ & 29.4 & 31.7 & 24.6 & 24.4 & 22.9 & 20.3 & 22.0 & 23.2 & 23.7 & 23.6 & 20 & 200 & 500 \\
\hline
\end{tabular}

Table 5

SOIL RESULTS FOR S21 $\div$ S30 SOIL SAMPLES FROM BUW 14 SITE

\section{Table 6}

SOIL RESULTS FOR S31 $\div$ S40 SOIL SAMPLES FROM BUW14

SITE 


\begin{tabular}{|c|c|c|c|c|c|c|c|c|c|c|c|c|c|}
\hline \multirow[t]{2}{*}{ Parameter } & \multirow[t]{2}{*}{ S4l } & \multirow[t]{2}{*}{$\mathrm{S} 42$} & \multirow[t]{2}{*}{$\mathrm{S} 43$} & \multirow[t]{2}{*}{ S44 } & \multirow[t]{2}{*}{ S45 } & \multirow[t]{2}{*}{ S46 } & \multirow[t]{2}{*}{ S47 } & \multirow[t]{2}{*}{$\mathrm{S} 48$} & \multirow[t]{2}{*}{ S49 } & \multirow[t]{2}{*}{$\mathrm{S} 50$} & \multicolumn{3}{|c|}{$\begin{array}{c}\text { Reference values from } \\
\text { Order } 756 / 1997, \\
\text { less sensinive land use }\end{array}$} \\
\hline & & & & & & & & & & & $\begin{array}{l}\text { Narmat } \\
\text { vahus }\end{array}$ & $\begin{array}{l}\text { Alme } \\
\text { Dordele }\end{array}$ & $\begin{array}{l}\text { Thammation } \\
\text { Merratele }\end{array}$ \\
\hline Dry matter (\%) & 91.2 & 95.2 & 93.3 & 95.7 & 95.4 & 94.4 & 93.4 & 95.5 & 93.9 & 88.7 & - & - & - \\
\hline Humus $(\% \mathrm{dm})$ & 1.90 & 5.09 & 5.01 & 4.79 & 3.99 & 4.23 & 3.94 & 2.23 & 2.12 & 3.57 & - & - & - \\
\hline $\begin{array}{l}\text { Kjeldahl Nitrogen } \\
(\% \mathrm{dm})\end{array}$ & 0.14 & 0.14 & 0.15 & 0.14 & 0.14 & 0.16 & 0.14 & 0.14 & 0.14 & 0.14 & - & - & - \\
\hline $\mathrm{Cu}(\mathrm{mg} / \mathrm{kg} \mathrm{dm})$ & 21.8 & 24.3 & 26.0 & 21.3 & 22.9 & 22.6 & 25.0 & 28.3 & 24.4 & 23.0 & 20 & 250 & 500 \\
\hline $\mathrm{Zn}(\mathrm{mg} / \mathrm{kg} \mathrm{dm})$ & 57.2 & 60.6 & 68.3 & 60.2 & 58.9 & 65.2 & 72.6 & 66.2 & 68.9 & 66.2 & 100 & 700 & 1,500 \\
\hline $\mathrm{Cr}(\mathrm{mg} / \mathrm{kg} \mathrm{dm})$ & 0.71 & 0.76 & 0.80 & 0.69 & 0.82 & 0.78 & 0.74 & 0.84 & 0.74 & 0.84 & 30 & 300 & 600 \\
\hline $\mathrm{Pb}(\mathrm{mg} / \mathrm{kg} \mathrm{dm})$ & 18.9 & 21.8 & 20.9 & 17.3 & 19.5 & 20.5 & 19.0 & 18.9 & 20.5 & 20.4 & 20 & 250 & 1,000 \\
\hline
\end{tabular}

Table 7

SOIL RESULTS

FOR S41 $\div$ S50

SOIL SAMPLES

FROM BUW14

SITE

\section{Table 8}

ANALITICAL RESULTS FOR GROUNDWATER SAMPLES

\begin{tabular}{|c|c|c|c|}
\hline $\begin{array}{l}\text { Parameter } \\
\text { (measure unit) }\end{array}$ & Well 1 & Well 2 & $\begin{array}{c}\text { Reference values } \\
\text { [16] }\end{array}$ \\
\hline $\mathrm{pH}$ (pH units) & 7.8 (measured at $20.2^{\circ} \mathrm{C}$ ) & 8.0 (measured at $20.1^{\circ} \mathrm{C}$ ) & $6.5 \div 9.5$ \\
\hline Conductivity $\left(\mu \mathrm{S} \mathrm{cm}^{-1}\right)$ & 642 (measured at $20.2^{\circ} \mathrm{C}$ ) & 614 (measured at $20.1^{\circ} \mathrm{C}$ ) & 2,500 \\
\hline Hardness (german degree) & 16.5 & 19.4 & $\min .5$ \\
\hline Total nitrogen $(\mathrm{mg} / \mathrm{l})$ & 5.21 & 8.38 & n.a. \\
\hline Nitrogen $(\mathrm{mg} / \mathrm{l})$ & 19.2 & 31.4 & 50 \\
\hline Nitrates (mg/) & 0.069 & 0.158 & 0.5 \\
\hline Ammonium (mg/l) & 0.071 & 0.149 & 0.5 \\
\hline Sulphates (mg/l) & 185 & 167 & 250 \\
\hline $\mathrm{COD}\left(\mathrm{mgO}_{2} / \mathrm{l}\right)$ & 3.21 & 3.07 & 5 \\
\hline $\mathrm{Pb}(\mathrm{mg} / \mathrm{l})$ & 0.008 & 0.006 & 0.01 \\
\hline $\mathrm{Cu}(\mathrm{mg} / \mathrm{l})$ & 0.011 & 0.007 & 0.10 \\
\hline $\mathrm{Zn}(\mathrm{mg} / \mathrm{l})$ & 0.049 & 0.268 & 5 \\
\hline $\mathrm{Cr}(\mathrm{mg} / \mathrm{l})$ & 0.001 & 0.001 & 0.05 \\
\hline $\mathrm{Ni}(\mathrm{mg} / \mathrm{l})$ & 0.01 & 0.01 & 0.02 \\
\hline $\mathrm{Fe}(\mathrm{mg} / \mathrm{l})$ & 0.189 & 0.195 & 0.20 \\
\hline
\end{tabular}

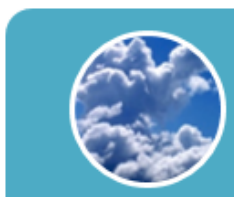

air quality status
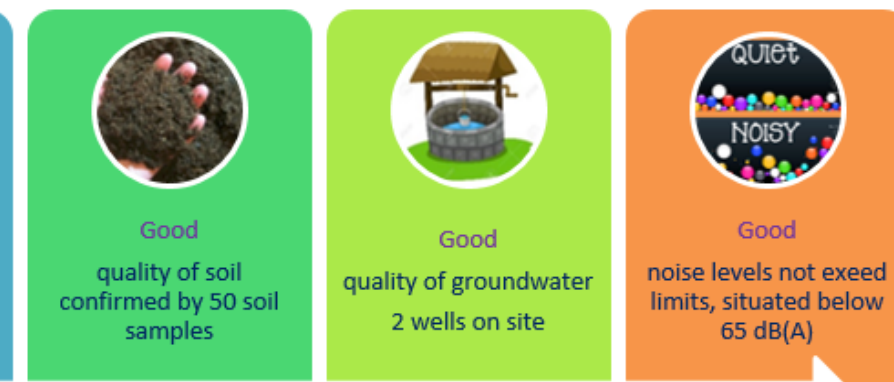

Assessment of site BUW 14 site reveal a good quality of the environment
Interpretation of the test results obtained for the 50 soil samples collected from the BUW14 site was performed according to in force Romanian legislation [15], taken into consideration reference values for chemical traces in the soil for less sensitive use of land, as it correlates with the commercial use of the area to be built here.

The results reveal that the soil samples quality is not affected by the pollution.

Regarding the other quality analyzed parameters, not regulated by the Order 756/1997 [15], it was found that: $\mathrm{pH}$ regime of the soil was in the range neutral to low alkaline; content of organic matter (humus) values were ranging from 0.65 to $4.76 \%$ d.m., an average value of $2.99 \%$ d.m. was found, which is consistent with the agricultural use of the analyzed land; organic nitrogen content expressed by the Kjeldahl Nitrogen indicator, highlights that the obtained values are for agricultural land, with no negative effect to the soil quality.

\section{Groundwater quality}

The analytical results obtained for groundwater are presented in table 8 , the results were compared with the reference values in accordance with Romanian legislation
[16] and the analyzed parameters fully meet the quality conditions of drinking water.

\section{Conclusions}

The assessment of the environmental components, in order to determine the quality of a BUW $14-10$ ha area on which an investment project will be developed, was made to provide the relevant environmental information before the project itself will be carried out. Also, there are all necessary assurances that the land purchased as a destination for logistic constructions was not previously affected by the pollution or the agricultural activities carried out on it. The assessment was carried out by air, soil, groundwater and noise level measurements and analysis, and objectively established that it was a unpolluted land, being certain premises for the development of the environmentunder the best environmental conditions. The general conclusions were synthesized in the form of a diagram, presented in figure 5 .

All the relevant environmental aspects of the BUW 14 site evaluation have provided clear premisis without additional environmental costs to the owner, in order to be able to develop in optimal conditions his new projects that 
includes logistics and office building in best quality environment conditions.

This site evaluation, with a pronounced applicative character, reveals once again how important is to assess the environment before implementing a new project. The evaluation costs are much smaller and easier for an owner than in the situation where a new project is developed without knowing the environmental conditions. The site may have a residual contamination of an previous site activity and could, in the absence of assessment, lead to environmental obligations that may affectnew investment projects.

\section{References}

1. SONAL, S.V., BIRVA D., J. Int. Environ. Appl. Sci., 3, no.5, 2008, p.358.

2. TATAR, A. M., Rev.Chim (Bucharest), 69, no.6, 2018, p.1403.

3. BALACEANU, C. M., MITIU, M.A., MARCUS, M.I., MINCU, M., Rev.Chim (Bucharest), 69, no.2, 2018, p.350.

4. TATAR, A.M., Rev.Chim (Bucharest), 69, no.5, 2018, p.1075.

5. STANESCU, B, KIM, L, CUCIUREANU, A, LEHR, C B, $19^{\text {th }}$ International Symposium The Environment and the Industry, Proceedings Book, 2016, p. 112.

6. SCRADEANU, M, IORDACHE, V, PALCU, M, SCARDEANU, D, $19^{\text {th }}$ International Symposium The Environment and the Industry, Proceedings Book, 2016, p. 292.
7. PETRESCU, M., BUCUR, E, DIODIU, R, BRATU, M, SERBANESCU, A, BARBU, $M, 20^{\text {th }}$ International Symposium The Environment and the Industry, Proceedings Book, 2017, p. 213.

8. DANCIULESCU, V., BUCUR, E, PASCU, L F, VASILE, A, , J. Environ. Prot. Ecol., 16, no. 3, 2015. p.815.

9. *** https://trans.info/ro/piata-de-logistica-din-romania-atins-un-nivelrecord-2017-77463, in Romanian

10. *** LAW 104, 15J une 2011 regarding air quality. Romanian's Official Monitor, Part.1, no.452, 28 J une 2011.

11. *** STAS 12574-87, Air in protected areas. Quality conditions, in Romanian.

12. *** STAS 10009-2017 Acoustics. Permissible limits of ambient noise level, in Romanian

13. *** ISO 18400-102:2017 - Soil Quality. Sampling. Part 102: Selection and application of sampling techniques.

14. *** ISO 10381-5:2005 - Soil Quality. Sampling. Part 5. Guidance on the procedure for investigating soil contamination in urban and industrial sites.

15. *** ORDER 756/1997, for the approval of the Regulation regardind the environmental pollution assessment, with subsequent modifications and completions, Romanian's Official Monitor, no.3030 bis, 6 November, 1997.

16. *** LAW 458, 2002 ( $\mathrm{rl}$ ) regarding drinking water quality. Romanian's Official Monitor, Part.1, no.58, update 24 January 2012.

Manuscript received: 17.08 .2018 